\title{
Unifying Strongly Clean Power Series Rings
}

\author{
Alexander J. Diesl Daniel R. Shifflet
}

February 2, 2018

\begin{abstract}
It is unknown whether a power series ring over a strongly clean ring is, itself, always strongly clean. Although a number of authors have shown that the above statement is true in certain special cases, the problem remains open, in general. In this article, we look at a class of strongly clean rings, which we call the optimally clean rings, over which power series are strongly clean. This condition is motivated by work in [DDGK12] and [DDI $\left.{ }^{+} 13\right]$. We explore the properties of optimally clean rings and provide many examples, highlighting the role that this new class of rings plays in investigating the question of strongly clean power series.
\end{abstract}

\section{Introduction}

Following [Nic99], an element $a$ of a ring $R$ is called strongly clean if there is an idempotent $e \in R$ such that $a e=e a$ and such that $a-e$ is a unit in $R$. A ring is called strongly clean if every one of its elements admits a strongly clean decomposition. In keeping with the philosophy of [Nic99], we view the strongly clean rings as a generalization of the classical strongly $\pi$-regular rings. In addition to the strongly $\pi$-regular rings, there are many other examples of strongly clean rings, including local rings, commutative exchange rings, and the ring of continuous functions on a strongly zero-dimensional space. Moreover, many authors over the last decade have studied the stability of the strongly clean property under certain ring extensions (e.g. full and triangular matrix rings, power series rings, group rings, etc.), thus adding substantially to the stock of examples. Despite this large collection of examples, some basic questions about strongly clean rings, many motivated by known results about strongly $\pi$-regular rings, remain unanswered. For this reason, the creation and study of new classes of strongly clean rings remains important. This provides one of our primary motivations for studying strongly clean power series rings.

Although many articles have been written about strongly clean full matrix rings and triangular matrix rings, relatively little attention has been given to the power series case in its own right (though, as we shall see, mentions of strongly clean power series rings are scattered throughout the literature). In the present article, we aim to fill this void by focusing exclusively on strongly clean power series rings, with the goal of unifying and extending what is currently known. To set the stage, we summarize the progress to date. In [CZ07, Theorem 2.1 , it is shown that any (skew) power series over a strongly $\pi$-regular ring is strongly clean. In [CWZ09, Theorem 20], a similar set of ideas is used to show an analogous result in the 
uniquely strongly clean case. Finally, in [DDGK12], the problem of strongly clean power series is viewed from the more general perspective of rings which are complete with respect to an ideal. Using this viewpoint, the authors give a streamlined proof of the result in [CZ07] and identify a condition (see Theorem 2.1) which is strictly weaker than the strongly $\pi$-regular condition, yet which still implies that power series over the ring are strongly clean. It is this condition that serves as the starting point for our work in the present article.

To date, no one has yet determined whether the power series ring over a strongly clean ring is, itself, strongly clean, nor has anyone even established necessary and sufficient conditions that characterize rings that have strongly clean power series rings. These open problems motivate the current investigation. In Section 2, we define the optimally clean elements and show (through a specialization of results in [DDGK12] and [DDI $\left.{ }^{+} 13\right]$ ) that a power series is strongly clean if its constant term is optimally clean. The remainder of Section 2 is devoted to an investigation of the basic properties of optimally clean elements and rings.

In Section 3, we devote our attention to the construction of examples of optimally clean rings. One important question about the optimally clean condition is whether it is necessary for the formation of a strongly clean power series ring. As was mentioned earlier, the literature on strongly clean rings contains numerous examples of strongly clean power series rings. However, each of these examples is investigated by ad hoc methods specific to the particular article in which it appears. In Section 3, we show that many known examples of strongly clean rings are optimally clean, thereby unifying all of the previously-mentioned strongly

clean power series examples using the optimally clean property. We close the section with an example of a strongly clean element which is not optimally clean.

Our ring-theoretic conventions will generally follow those in [Lam01]. All rings are assumed to be associative with identity, which is preserved by homomorphisms. We use $J(R)$, $U(R)$, and $Z(R)$ to denote, respectively, the Jacobson radical, the group of units, and the center of a ring $R$. If $a$ is an element of a ring $R$, then $r_{a}$ and $l_{a}$ will denote, respectively, right and left multiplication by $a$. As we will be working extensively with power series, we will assume (unless otherwise noted) that power series are written with coefficients on the left. Finally, although the main aim of the article is the study of power series rings, many of our results are also valid for certain skew power series rings, and we will present results in this more general framework whenever it is convenient to do so.

\section{Strongly Clean Skew Power Series Rings}

We begin by recalling two results about strongly clean elements in rings that are complete with respect to an ideal. The first theorem is one of the main results of [DDGK12]. Note that we have changed the notation slightly from the original. This has been done to ensure consistency with the other results in the present article.

Theorem 2.1. ([DDGK12, Theorem 8]) Let $R$ be a ring that is complete with respect to an ideal $I$. For each $m$, let $\pi_{m}: R \rightarrow R / I^{m}$ denote the natural quotient map. Let $a \in R$, and suppose that there is an element $e \in R$ such that $\pi_{1}(e)$ is an idempotent in $\pi_{1}(R)$ and such that $\pi_{1}(a)$ is strongly clean with respect to $\pi_{1}(e)$. Let $f \in R$ be any idempotent which agrees with e, modulo $I$, and let $f^{\prime}=1-f$. Suppose that for every such idempotent $f$ and for every 
$m \geq 1$, the maps

$$
l_{\pi_{m}(f a f)}-r_{\pi_{m}\left(f^{\prime} a f^{\prime}\right)}: \pi_{m}(f)\left(R / I^{m}\right) \pi_{m}\left(f^{\prime}\right) \rightarrow \pi_{m}(f)\left(R / I^{m}\right) \pi_{m}\left(f^{\prime}\right)
$$

and

$$
l_{\pi_{m}\left(f^{\prime} a f^{\prime}\right)}-r_{\pi_{m}(f a f)}: \pi_{m}\left(f^{\prime}\right)\left(R / I^{m}\right) \pi_{m}(f) \rightarrow \pi_{m}\left(f^{\prime}\right)\left(R / I^{m}\right) \pi_{m}(f)
$$

are surjective and have the additional property that each element of $\pi_{m}\left(I^{m-1}\right)$ has a preimage in $\pi_{m}\left(I^{m-1}\right)$. Then a is strongly clean.

The second result is a slightly different formulation of the same idea, proved recently in $\left[\mathrm{DDI}^{+} 13\right]$ (as in Theorem 2.1, the notation has been changed slightly from the original for the sake of consistency).

Theorem 2.2. ([DDI+ 13, Theorem 4.3]) Let $R$ be a ring that is complete with respect to an ideal $I$. For each $m$, let $\pi_{m}: R \rightarrow R / I^{m}$ be the natural quotient map. Let $a \in R$, and suppose that $e$ is an idempotent of $R$ such that $\pi_{1}(a)$ is strongly clean with respect to $\pi_{1}(e)$. Writing $e^{\prime}=1-e$, suppose further that the maps

$$
l_{\pi_{1}(e a e)}-r_{\pi_{1}\left(e^{\prime} a e^{\prime}\right)}: e\left(I^{m} / I^{m+1}\right) e^{\prime} \rightarrow e\left(I^{m} / I^{m+1}\right) e^{\prime}
$$

and

$$
l_{\pi_{1}\left(e^{\prime} a e^{\prime}\right)}-r_{\pi_{1}(e a e)}: e^{\prime}\left(I^{m} / I^{m+1}\right) e \rightarrow e^{\prime}\left(I^{m} / I^{m+1}\right) e
$$

are surjective for each $m \geq 0$. Then a is strongly clean in $R$ with respect to an idempotent $f$ such that $f-e \in I$.

Note that Theorem 2.1 and Theorem 2.2 bear a strong resemblance to each other. Before proceeding further, we will sketch a proof that they are, in fact, equivalent. Suppose first that we have a ring $R$, complete with respect to an ideal $I$, and an element $a \in R$ for which the hypotheses of Theorem 2.1 hold. By completeness, we can lift $\pi_{1}(e)$ to an actual idempotent in $R$. We may thus assume that the element $e$, given in Theorem 2.1, is idempotent. We then claim that the maps given in the hypotheses of Theorem 2.2 are surjective. We fix $m$ and consider the first of the two maps. Choose an arbitrary element $z \in e I^{m} e^{\prime}$. By the hypotheses of Theorem 2.1, there is an element $y \in I^{m}$ such that

$$
(\text { eae })\left(e y e^{\prime}\right)-\left(e y e^{\prime}\right)\left(e^{\prime} a e^{\prime}\right)-z \in I^{m+1}
$$

It is then straightforward to verify that

$$
\pi_{1}(e a e) \pi_{m+1}\left(e y e^{\prime}\right)-\pi_{m+1}\left(e y e^{\prime}\right) \pi_{1}\left(e^{\prime} a e^{\prime}\right)=\pi_{m+1}(z) .
$$

Thus, $\pi_{m+1}\left(e y e^{\prime}\right) \in e\left(I^{m} / I^{m+1}\right) e^{\prime}$ maps to $\pi_{m+1}(z)$ under the map in question. The surjectivity of the second map (for every $m$ ) is proved analogously.

On the other hand, suppose now that we have a ring $R$, an ideal $I$ and an element $a$ as before, but assume instead that the hypotheses of Theorem 2.2 hold. Let $f \in R$ be any idempotent such that $f-e \in I$. We claim that all of the maps in Theorem 2.1 are surjective. As before, we focus on the first map, the argument for the second being similar. The proof will proceed by induction on $m$. The base case, $m=1$, is immediate. Suppose now that 
$l_{\pi_{m}(f a f)}-r_{\pi_{m}\left(f^{\prime} a f^{\prime}\right)}$ is surjective for $m=k$. We claim that the same is true for $m=k+1$. Let $z \in f R f^{\prime}$ be arbitrary. By induction, we know that there is an element $y^{\prime} \in R$ such that

$$
(f a f)\left(f y^{\prime} f^{\prime}\right)-\left(f y^{\prime} f^{\prime}\right)\left(f^{\prime} a f^{\prime}\right)-z \in f I^{k} f^{\prime} .
$$

Using the surjectivity of the first map in the hypotheses of Theorem 2.2 (observing that the maps in the statement of Theorem 2.2 are unaffected by replacing $e$ with $f$, since $e$ and $f$ are equal modulo $I$ ), there is then an element $y_{k} \in I^{k}$ such that

$$
(f a f)\left(f y_{k} f^{\prime}\right)-\left(f y_{k} f^{\prime}\right)\left(f^{\prime} a f^{\prime}\right)-\left[z-(f a f)\left(f y^{\prime} f^{\prime}\right)+\left(f y^{\prime} f^{\prime}\right)\left(f^{\prime} a f^{\prime}\right)\right] \in I^{k+1} .
$$

Rewriting this gives

$$
(f a f)\left(f y^{\prime} f^{\prime}+f y_{k} f^{\prime}\right)-\left(f y^{\prime} f^{\prime}+f y_{k} f^{\prime}\right)\left(f^{\prime} a f^{\prime}\right)-z \in I^{k+1},
$$

which shows that $\pi_{k+1}\left(f y^{\prime} f^{\prime}+f y_{k} f^{\prime}\right)$ maps to $\pi_{k+1}(z)$ under the map in question. This completes our induction. Note finally that the condition on the preimage of $\pi_{k+1}\left(I^{k}\right)$, required in Theorem 2.1, is also satisfied. Indeed, if $z \in I^{k}$, we may take $y^{\prime}=0$ in the above argument.

Specializing to the case of a skew power series ring, we extract the following corollary.

Corollary 2.3. Let $R$ be a ring, and let $\sigma$ be a ring endomorphism of $R$. Let $a=\sum_{i \geq 0} a_{i} x^{i} \in$ $R[[x ; \sigma]]$, and suppose that there is an idempotent $e_{0} \in R$, with respect to which $a_{0}$ is strongly clean. Suppose further that for every $n \geq 0$, the maps (writing $f_{0}=1-e_{0}$ )

$$
l_{e_{0} a_{0} e_{0}}-r_{\sigma^{n}\left(f_{0} a_{0} f_{0}\right)}: e_{0} R \sigma^{n}\left(f_{0}\right) \rightarrow e_{0} R \sigma^{n}\left(f_{0}\right)
$$

and

$$
l_{f_{0} a_{0} f_{0}}-r_{\sigma^{n}\left(e_{0} a_{0} e_{0}\right)}: f_{0} R \sigma^{n}\left(e_{0}\right) \rightarrow f_{0} R \sigma^{n}\left(e_{0}\right)
$$

are surjective. Then a is strongly clean in $R[[x ; \sigma]]$.

Proof. Apply Theorem 2.2 directly to the ring $R[[x ; \sigma]]$, which is complete with respect to the ideal $I=(x)$. Note that, in this situation, the bimodule $I^{n} / I^{n+1}$ is precisely $R$ itself, except that the right multiplication has been twisted by $\sigma^{n}$.

The hypotheses of Corollary 2.3 give the precise condition with which we will concern ourselves. We make the following definition.

Definition 2.4. Let $R$ be a ring, and let $\sigma$ be a ring endomorphism of $R$. An element $a \in R$ is called $\sigma$-optimally clean if there exists an idempotent $e \in R$ with respect to which $a$ is strongly clean and with the further property that for every integer $n \geq 0$, the maps (writing $f=1-e)$

$$
l_{\text {eae }}-r_{\sigma^{n}(f a f)}: e R \sigma^{n}(f) \rightarrow e R \sigma^{n}(f)
$$

and

$$
l_{f a f}-r_{\sigma^{n}(e a e)}: f R \sigma^{n}(e) \rightarrow f R \sigma^{n}(e)
$$

are surjective. In the case that $\sigma$ is the identity, we will refer to such an element as optimally clean. A ring will be called $\sigma$-optimally clean (respectively, optimally clean) if each of its elements is $\sigma$-optimally clean (respectively, optimally clean). If an element or a ring is $\sigma$-optimally clean for every endomorphism $\sigma$, we will say that it is skew optimally clean. 
Remark 2.5. The term "optimally clean" was initially motivated by the situation in the strongly $\pi$-regular case. As we will see in Proposition 3.5, a strongly $\pi$-regular element is optimally clean with respect to the idempotent which witnesses the strong $\pi$-regularity. This idempotent is "optimal" in the sense that it is the smallest idempotent (in the usual partial order on idempotents) with respect to which the element in question is strongly clean. As we will see as we explore more examples in Section 3, many constructions of strongly clean decompositions involve picking an idempotent which is "best" in some sense, and such choices often produce idempotents that yield optimally clean decompositions.

Before proceeding further, we would like to explicitly point out that there are several slightly different, but equivalent, formulations of Definition 2.4. The first concerns the map $l_{\text {eae }}$. Since $a$ and $e$ commute and since the domain of this map is $e R \sigma^{n}(f)$, we can safely replace "l $l_{e a e}$ " with " $l_{a}$ " (which, while shorter, does not make it as clear that we are fundamentally working with Peirce corners). Analogous substitutions can be made for the other maps in Definition 2.4. Second, although Definition 2.4 relies ostensibly on the surjectivity of two maps, these two maps can actually be combined (via a direct sum) into the single map

$$
l_{a}-r_{\sigma^{n}(a)}:\left(l_{e}-r_{\sigma^{n}(e)}\right)^{2} R \rightarrow\left(l_{e}-r_{\sigma^{n}(e)}\right)^{2} R .
$$

Finally, the next result gives a third alternate characterization, which omits reference to the Peirce corners entirely. The following notation will be useful. If $\sigma$ is a ring endomorphism of $R$, then we will define the $\sigma$-commutator of $r$ and $s$ as follows: $[r, s]_{\sigma}=r s-s \sigma(r)$.

Proposition 2.6. Suppose that $R$ is a ring, and suppose that $\sigma$ is a ring endomorphism of $R$. Let $a \in R$, and suppose that $a=e+u$ is a strongly clean decomposition of $a$. The following are equivalent.

(1) The element a is $\sigma$-optimally clean with respect to $e$.

(2) For every $n \geq 0$ and for every $b \in R$, there is an element $y \in R$ such that $[a, y]_{\sigma^{n}}=$ $[e, b]_{\sigma^{n}}$.

Proof. Suppose first that $a$ is $\sigma$-optimally clean with respect to $e$. Write $f=1-e$. Let $n \geq 0$ and let $b \in R$. Since $a$ is $\sigma$-optimally clean, we can find elements $w \in e R \sigma^{n}(f)$ and $z \in f R \sigma^{n}(e)$ such that $(e a e) w-w \sigma^{n}(f a f)=e b \sigma^{n}(f)$ and $(f a f) z-z \sigma^{n}(e a e)=f b \sigma^{n}(e)$. Let $y=w-z$. We claim that $[a, y]_{\sigma^{n}}=[e, b]_{\sigma^{n}}$. Noting that $a=e a e+f a f$, we see that

$$
\begin{aligned}
{[a, y]_{\sigma^{n}} } & =[e a e+f a f, w-z]_{\sigma^{n}} \\
& =(e a e) w-(f a f) z-w \sigma^{n}(f a f)+z \sigma^{n}(e a e) \\
& =e b \sigma^{n}(f)-f b \sigma^{n}(e) \\
& =e b \sigma^{n}(f)+e b \sigma^{n}(e)-e b \sigma^{n}(e)-f b \sigma^{n}(e) \\
& =e b-b \sigma^{n}(e) \\
& =[e, b]_{\sigma^{n}} .
\end{aligned}
$$

The reverse implication is proved similarly. 
Though we will primarily use the original definition of the optimally clean property (as stated in Definition 2.4), we will feel free to use any of the other characterizations, should it be more suitable in a particular situation.

Returning now to the main thread of the investigation, the following corollary is immediate.

Corollary 2.7. Let $R$ be a ring, and let $\sigma$ be a ring endomorphism of $R$. Let $a=\sum_{i \geq 0} a_{i} x^{i} \in$ $R[[x ; \sigma]]$. If $a_{0}$ is $\sigma$-optimally clean in $R$, then a is strongly clean in $R[[x ; \sigma]]$. If $R$ is $\sigma$-optimally clean, then $R[[x ; \sigma]]$ is strongly clean.

Remark 2.8. Now that we have established the optimally clean property, we can formally pose several questions about it. First among these is whether or not a strongly clean ring is always optimally clean. This question can be broken into two sub-questions, the first being whether or not the strongly clean condition passes to power series rings, and the second being whether a strongly clean power series ring necessitates an optimally clean base ring. These are the fundamental issues that guide this investigation.

We begin by exploring some of the basic properties of the $\sigma$-optimally clean property.

Proposition 2.9. (1) Let $R$ be a ring, and let $\sigma$ be a ring endomorphism of $R$ such that $R$ is $\sigma$-optimally clean. Let $I$ be an ideal of $R$ such that $\sigma(I) \subseteq I$, and let $\bar{\sigma}$ be the induced endomorphism on $\bar{R}=R / I$. Then $\bar{R}$ is $\bar{\sigma}$-optimally clean.

(2) Let $\left\{R_{\alpha}\right\}_{\alpha \in A}$ be a collection of rings. For each $\alpha \in A$, let $\sigma_{\alpha}$ be a ring endomorphism of $R_{\alpha}$. Let $R=\prod R_{\alpha}$, and $\sigma=\left(\sigma_{\alpha}\right)$. If $R_{\alpha}$ is $\sigma_{\alpha}$-optimally clean for each $\alpha \in A$, then $R$ is $\sigma$-optimally clean.

Proof. These are straightforward verifications.

It is well known that the strongly clean condition passes to Peirce corners (this was proved independently by T.Y. Lam and E. Sanchez-Campos; proofs also appear in [Che06] and $[\mathrm{CYZ06a}])$. Our next result shows that this is true for the optimally clean condition as well.

Proposition 2.10. If $R$ is optimally clean, then eRe is optimally clean for any $e^{2}=e \in R$.

Proof. Suppose that $R$ is optimally clean, and let $e \in R$ be any idempotent. Let $e r e \in e R e$, and let $f \in R$ be an idempotent with respect to which ere is optimally clean. Since ere is strongly clean with respect to $f$, it is known that $f=e f e \in e R e$ and that $f-e r e \in U(e R e)$ (this basic fact underlies all of the proofs that strong cleanness passes to corners; see [Die13, Proposition 2.9] for a general statement and proof). We claim now that ere is optimally clean with respect to $f$, in $e R e$. Let eze be an arbitrary element of $e R e$. Since ere is optimally clean with respect to $f$, in $R$, there is an element $y \in R$ such that [ere,y] $=[f$,eze $]$. But then it is immediate that $[e r e, e y e]=[f, e z e]$. Thus, $e R e$ is optimally clean.

Remark 2.11. Indeed, if $R$ is $\sigma$-optimally clean for some $\sigma$, and if $e \in R$ is an idempotent such that $\sigma(e)=e$, then Proposition 2.10 can be easily extended (with the same proof) to show that $e R e$ is $\sigma$-optimally clean as well. 


\section{Examples of $\sigma$-Optimally Clean Rings}

Having established the basic properties of $\sigma$-optimally clean rings in the previous section, we will now give examples of such rings. We will show that a wide variety of strongly clean rings are optimally clean. As mentioned in the introduction, there are many results scattered throughout the literature that give examples of strongly clean power series rings. We will show that virtually every one of these examples can be viewed as a consequence of the relevant ring being optimally clean. Thus, the optimally clean notion unifies all of this work under a single idea.

To begin, it is clear that any power series over an abelian clean ring is strongly clean (an idempotent which witnesses the strong cleanness of the constant term works for the entire power series in this case). Moreover, it is shown in [CZ07, Theorem 2.1] and [DDGK12, Theorem 5] that any skew power series ring over a strongly $\pi$-regular ring is strongly clean. Our first few results reinterpret these facts from the $\sigma$-optimally clean perspective.

Proposition 3.1. Let $R$ be a ring with a ring endomorphism $\sigma$, and let $a \in R$. If there exists a central idempotent $e$ such that $a-e \in U(R)$ and $\sigma(e)=e$, then a is $\sigma$-optimally clean.

Proof. Note that, for any $b \in R$ and for any $n \geq 0,[e, b]_{\sigma^{n}}=e b-b \sigma^{n}(e)=0=[a, 0]_{\sigma^{n}}$.

Corollary 3.2. If $R$ is an abelian clean ring and $\sigma$ is a ring endomorphism of $R$ that fixes all of the idempotents of $R$, then $R$ is $\sigma$-optimally clean. In particular, every abelian clean ring is optimally clean.

Remark 3.3. By Proposition 3.1, any unit, nilpotent or element of the Jacobson radical is skew optimally clean, since each of these elements is strongly clean with respect to either 1 or 0. By Corollary 3.2, every uniquely clean ring (defined in [NZ04]) is optimally clean.

The next result follows directly from Corollary 3.2.

Proposition 3.4. Every local ring is skew optimally clean.

Recall that an element $a$ of a ring $R$ is called strongly $\pi$-regular if there is an idempotent $e \in R$, commuting with $a$, such that $a-e$ is invertible and $e a$ is nilpotent. As part of their proof of Theorem 2.1, the authors of [DDGK12] show that every strongly $\pi$-regular ring is skew optimally clean. For completeness, we give a quick argument here.

Proposition 3.5. Let $R$ be a ring, and let $a \in R$. If a is strongly $\pi$-regular, then $a$ is skew optimally clean. Consequently, any strongly $\pi$-regular ring is skew optimally clean.

Proof. Let $\sigma$ be any ring endomorphism of $R$, and let $a \in R$. Since $a$ is strongly $\pi$-regular, there is an idempotent $e \in R$ such that $a$ is strongly clean with respect to $e$ and such that eae is nilpotent. We claim that $a$ is $\sigma$-optimally clean with respect to $e$. Let $e b \sigma^{n}(f)$ be an arbitrary element of $e R \sigma^{n}(f)$. Note that $f a f \in U(f R f)$, since $a$ is strongly clean with respect to $e$. Let

$$
y=-\sum_{i=1}^{k}(e a e)^{i-1}\left(e b \sigma^{n}(f)\right) \sigma^{n}(f a f)^{-i}
$$


where $k$ is the index of nilpotence of eae and where the inverse of $f a f$ is taken in $f R f$. Then $y \in e R \sigma^{n}(f)$, and $(e a e) y-y \sigma^{n}(f a f)=e b \sigma^{n}(f)$, which shows that the map $l_{e a e}-r_{\sigma^{n}(f a f)}$ is surjective. The surjectivity of $l_{f a f}-r_{\sigma^{n}(e a e)}$ follows similarly.

Remark 3.6. Proposition 3.5 implies that every idempotent is skew optimally clean.

Having shown that the $\sigma$-optimally clean condition recovers the basic results about strongly clean power series rings, we will now investigate the behavior of the $\sigma$-optimally clean condition with respect to some of the ring extensions that are most often studied in conjunction with strongly clean rings. We start with a very general result, which will have relevance for both power series rings and triangular matrix rings.

Lemma 3.7. Let $R$ be a ring, and let $I$ be an ideal of $R$, with respect to which $R$ is complete. Use "bar" notation to denote passage to the quotient $R / I$. Let $\sigma$ be a ring endomorphism of $R$. Let $a \in R$, and suppose that there is an idempotent $e \in R$ with respect to which a is strongly clean. Let $f=1-e$. Finally, suppose that for every $k \geq 0$, the maps

$$
l_{\overline{e a e}}-r_{\overline{\sigma(f a f)}}: e\left(I^{k} / I^{k+1}\right) \sigma(f) \rightarrow e\left(I^{k} / I^{k+1}\right) \sigma(f)
$$

and

$$
l_{\overline{f a f}}-r_{\overline{\sigma(e a e)}}: f\left(I^{k} / I^{k+1}\right) \sigma(e) \rightarrow f\left(I^{k} / I^{k+1}\right) \sigma(e)
$$

are surjective. Then the maps

$$
l_{\text {eae }}-r_{\sigma(f a f)}: e R \sigma(f) \rightarrow e R \sigma(f)
$$

and

$$
l_{f a f}-r_{\sigma(e a e)}: f R \sigma(e) \rightarrow f R \sigma(e)
$$

are surjective.

Proof. We will show that the map on $e R \sigma(f)$ is surjective; the surjectivity of the map on $f R \sigma(e)$ follows by analogy. Let $e b \sigma(f) \in e R \sigma(f)$. We will construct a sequence $\left\{x_{i}\right\}$ of elements of $R$ such that

(1) $x_{i} \in e R \sigma(f)$ for each $i \geq 1$

(2) $(e a e) x_{i}-x_{i} \sigma(f a f)-e b \sigma(f) \in I^{i}$ for each $i \geq 1$

(3) $x_{i+1}-x_{i} \in I^{i}$ for each $i \geq 1$.

By completeness, we can take $x=\lim x_{i} \in e R \sigma(f)$. It is then immediate that (eae) $x-$ $x \sigma(f a f)=e b \sigma(f)$.

We construct the elements $x_{i}$ by induction. By hypothesis, there is an element $\overline{x_{1}} \in R / I$ such that $(\overline{e a e}) \overline{x_{1}}-\overline{x_{1}}(\overline{\sigma(f a f)})=\overline{e b f}$. We may also assure that $x_{1} \in e R \sigma(f)$, replacing $x_{1}$ by $e x_{1} \sigma(f)$, if necessary. Thus, we have a suitable element $x_{1}$.

Suppose now that elements $x_{1}, \ldots, x_{k}$ have been constructed as prescribed. In particular, we know that

$$
(e a e) x_{k}-x_{k} \sigma(f a f)-e b \sigma(f) \in I^{k}
$$


Let $\pi_{k+1}: R \rightarrow R / I^{k+1}$ be the natural quotient map. Then,

$$
\pi_{k+1}\left((e a e) x_{k}-x_{k} \sigma(f a f)-e b \sigma(f)\right) \in e\left(I^{k} / I^{k+1}\right) \sigma(f) .
$$

By hypothesis, there is an element $y_{k} \in e\left(I^{k} / I^{k+1}\right) \sigma(f)$ such that

$$
(\overline{e a e}) y_{k}-y_{k}(\overline{\sigma(f a f)})=\pi_{k+1}\left((e a e) x_{k}-x_{k} \sigma(f a f)-e b \sigma(f)\right) .
$$

Lift $y_{k}$ to an element $z_{k} \in e R \sigma(f)$ such that $\pi_{k+1}\left(z_{k}\right)=y_{k}$, and set $x_{k+1}=x_{k}-z_{k}$. Then it is easy to see that $x_{k+1}$ satisfies conditions (1), (2) and (3). This completes the induction and the proof.

Lemma 3.7 leads immediately to the following useful result.

Theorem 3.8. Suppose that $R$ is a ring complete with respect to an ideal I, and use "bar" notation to denote passage to the quotient $R / I$. Let $\sigma$ be a ring endomorphism of $R$. Let $a \in R$, and suppose that there is an idempotent $e \in R$ with respect to which a is strongly clean. Write $f=1-e$, and suppose further that for every $n \geq 0$ and every $k \geq 0$, the maps

$$
l_{\overline{\text { eae }}}-r_{\overline{\sigma^{n}(f a f)}}: e\left(I^{k} / I^{k+1}\right) \sigma^{n}(f) \rightarrow e\left(I^{k} / I^{k+1}\right) \sigma^{n}(f)
$$

and

$$
l_{\overline{f a f}}-r_{\overline{\sigma^{n}(e a e)}}: f\left(I^{k} / I^{k+1}\right) \sigma^{n}(e) \rightarrow f\left(I^{k} / I^{k+1}\right) \sigma^{n}(e)
$$

are surjective. Then a is $\sigma$-optimally clean.

Proof. We claim that $a$ is $\sigma$-optimally clean with respect to $e$. This follows by applying Lemma 3.7 to every power of $\sigma$.

Remark 3.9. Note that the hypotheses of Theorem 3.8 are very similar to those of both Theorem 2.1 and Theorem 2.2. As we will see further on, this will have implications for our search for a strongly clean ring that is not optimally clean.

Theorem 3.8 can be applied to both power series rings and triangular matrix rings. We begin with the power series case. Before stating our next result, a comment about iterated skew power series is in order. Suppose that $R$ is a ring and that $\sigma$ and $\tau$ are two ring endomorphisms of $R$. If $\sigma$ and $\tau$ commute, then we can extend $\sigma$ to an endomorphism of $R[[y ; \tau]]$ by defining $\sigma\left(\sum a_{i} y^{i}\right)=\sum \sigma\left(a_{i}\right) y^{i}$. This allows for the construction of the iterated skew power series $(R[[y ; \tau]])[[x ; \sigma]]$.

Theorem 3.10. Let $R$ be a ring, and let $\sigma$ and $\tau$ be two commuting ring endomorphisms of $R$. Extend $\sigma$ to a ring endomorphism of $R[[y ; \tau]]$ as discussed above. Suppose that for each $a_{0} \in R$ there is an idempotent $e_{0} \in R$ with respect to which $a_{0}$ is $\sigma^{i} \tau^{j}$-optimally clean for every $i, j \geq 0$. Then $R[[y ; \tau]]$ is $\sigma$-optimally clean.

Proof. Let $a=\sum a_{i} y^{i}$ be an element of $R[[y ; \tau]]$. Fix an idempotent $e_{0}$ with respect to which $a_{0}$ is $\sigma^{i} \tau^{j}$-optimally clean for every $i, j \geq 0$. Since $a_{0}$ is $\tau$-optimally clean, Corollary 2.7 and Theorem 2.2 guarantee that there is an idempotent $e=\sum_{i} e_{i} y^{i} \in R[[y ; \tau]]$, whose constant term is $e_{0}$, with respect to which $a$ is strongly clean. We claim that $a$ is, in fact, $\sigma$-optimally 
clean with respect to $e$. Let $f_{0}=1-e_{0}$. Translating Theorem 3.8 to the skew power series case, we must show that, for each $n \geq 0$ and each $k \geq 0$, the maps

$$
l_{e_{0} a_{0} e_{0}}-r_{\sigma^{n}\left(f_{0} a_{0} f_{0}\right)}: e_{0} R\left(\tau^{k}\right) \sigma^{n}\left(f_{0}\right) \rightarrow e_{0} R\left(\tau^{k}\right) \sigma^{n}\left(f_{0}\right)
$$

and

$$
l_{f_{0} a_{0} f_{0}}-r_{\sigma^{n}\left(e_{0} a_{0} e_{0}\right)}: f_{0} R\left(\tau^{k}\right) \sigma^{n}\left(e_{0}\right) \rightarrow f_{0} R\left(\tau^{k}\right) \sigma^{n}\left(e_{0}\right)
$$

are surjective, where $R\left(\tau^{k}\right)$ is the $(R, R)$-bimodule whose underlying abelian group is $R$, whose left $R$-action is usual multiplication in $R$ and whose right $R$-action is "twisted" by $\tau^{k}$ (i.e. $r \cdot s=r \tau^{k}(s)$ ). By hypothesis, $a_{0}$ is $\sigma^{n} \tau^{k}$-optimally clean with respect to $e_{0}$, which implies that the maps above are surjective. By Theorem $3.8, R[[y ; \tau]]$ is $\sigma$-optimally clean.

Specializing Theorem 3.10 to the case where each of $\sigma$ and $\tau$ is the identity, we get the following.

Corollary 3.11. Let $R$ be a ring. The following are equivalent.

(1) $R$ is optimally clean.

(2) $R[[x]]$ is optimally clean.

We can also apply Theorem 3.8 to triangular matrix rings. Strongly clean triangular matrix rings have been investigated by a number of authors (e.g. [CYZ06a], [BDD07], $\left.\left[\mathrm{DDI}^{+} 13\right]\right)$. In many such articles, the authors use their respective results and techniques to show that triangular matrices over certain power series rings are strongly clean. Our next series of results shows that the vast majority of these examples follow from the fact that the relevant underlying rings are $\sigma$-optimally clean for appropriate choices of $\sigma$.

We begin with a general observation about triangular matrix rings. Suppose that $R$ is a ring, fix a positive integer $n$, and let $S=\mathbb{T}_{n}(R)$ denote the ring of upper-triangular matrices over $R$. Suppose further that $\sigma$ is a ring endomorphism of $R$, and extend $\sigma$ componentwise to an endomorphism (which we will also call $\sigma$ ) of $\mathbb{T}_{n}(R)$. Let $I$ be the ideal of $S$ which consists of all upper-triangular matrices with zeroes along the diagonal. Note that $I^{n}=0$, so $S$ is complete with respect to $I$. Let $A=\left(a_{i j}\right)$ be a strongly clean element of $S$, and suppose that $E=\left(e_{i j}\right)$ is an idempotent with respect to which $A$ is strongly clean. Write $F=\left(f_{i j}\right)=1-E$. Preparing for an application of Theorem 3.8, note that $I^{k} / I^{k+1}$ is the $k^{\text {th }}$ superdiagonal (under the convention that the main diagonal is the "zeroth superdiagonal"). Under this identification, elements of $I^{k} / I^{k+1}$ can be represented as vectors $\left(b_{1, k+1}, b_{2, k+2}, \ldots, b_{n-k, n}\right)$ (this makes sense as long as $0 \leq k \leq n-1)$. Moreover, if $v=\left(b_{1, k+1}, b_{2, k+2}, \ldots, b_{n-k, n}\right)$ is an element of $I^{k} / I^{k+1}$, then, for every $m$, we can write $\left(l_{E A E}-r_{\sigma^{m}(F A F)}\right)(v)=\left(w_{1}, \ldots, w_{n-k}\right)$ where

$$
w_{i}=\left(e_{i i} a_{i i} e_{i i}\right) b_{i, k+i}-b_{i, k+i} \sigma^{m}\left(f_{k+i, k+i} a_{k+i, k+i} f_{k+i, k+i}\right) .
$$

Therefore, the map

$$
l_{E A E}-r_{\sigma^{m}(F A F)}: E\left(I^{k} / I^{k+1}\right) \sigma^{m}(F) \rightarrow E\left(I^{k} / I^{k+1}\right) \sigma^{m}(F)
$$


is surjective if the maps

$$
l_{e_{i i} a_{i i} e_{i i}}-r_{\sigma^{m}\left(f_{j j} a_{j j} f_{j j}\right)}: e_{i i} R \sigma^{m}\left(f_{j j}\right) \rightarrow e_{i i} R \sigma^{m}\left(f_{j j}\right)
$$

are surjective for every $1 \leq i \leq j \leq n$. The analogous implication holds for the map $l_{F A F}-r_{\sigma^{m}(E A E)}$. This discussion leads naturally to the following theorem.

Theorem 3.12. Fix a positive integer $n$. Suppose that $R$ is a ring with the property that, given any collection of $n$ elements $a_{1}, \ldots, a_{n} \in R$, there are idempotents $e_{1}, \ldots, e_{n} \in R$ such that $a_{i}$ is strongly clean with respect to $e_{i}$ for each $i$, and such that the maps

$$
\left(l_{a_{i}}-r_{a_{j}}\right):\left(l_{e_{i}}-r_{e_{j}}\right)^{2} R \rightarrow\left(l_{e_{i}}-r_{e_{j}}\right)^{2} R
$$

are surjective for every $1 \leq i \leq j \leq n$. Then $\mathbb{T}_{n}(R)$ is optimally clean.

Proof. Let $A=\left(a_{i j}\right) \in \mathbb{T}_{n}(R)$. Let $e_{11}, \ldots, e_{n n}$ be the idempotents of $R$ which correspond to the diagonal elements $a_{11}, \ldots, a_{n n}$ as indicated in the hypotheses. In order to apply Theorem 3.8, we must first construct an idempotent $E \in \mathbb{T}_{n}(R)$ with respect to which $A$ is strongly clean. Following the argument in $\left[\mathrm{DDI}^{+} 13\right.$, Theorem 2.5] (making the necessary minor changes), we see that our hypotheses imply that such an idempotent $E$ exists. Once we have the idempotent $E$, then the proof is completed by invoking Theorem 3.8, applied as indicated in the discussion preceding the current theorem.

Remark 3.13. Note that the existence of the idempotent $E$ in the first part of the proof of Theorem 3.12 follows also from either Theorem 2.1 or Theorem 2.2, appropriately specialized to the triangular matrix case.

Since the hypotheses of Theorem 3.12 are satisfied by a large number of the rings which are known to admit strongly clean triangular matrix rings, we immediately obtain a number of corollaries.

In [BDD07], the authors develop a set of conditions, denoted $\mathcal{B}_{n}$, and show that if $R$ is a local ring that satisfies condition $\mathcal{B}_{n}$, then $\mathbb{T}_{n}(R)$ is strongly clean. Essentially, the condition $\mathcal{B}_{n}$ is precisely the statement that the hypotheses of Theorem 3.12 are satisfied for a collection of $n$ elements of a local ring (see [BDD07, Theorem 22] for the full details). The following is immediate.

Corollary 3.14. Let $R$ be a local ring which satisfies the condition $\mathcal{B}_{n}$. Then $\mathbb{T}_{n}(R)$ is optimally clean.

As detailed in [BDD07], it is unknown whether the conditions $\mathcal{B}_{n}$ are necessary, in general, for the triangular matrix ring over a local ring to be strongly clean. It is known, however, that if $R$ is local, then $R$ satisfies $\mathcal{B}_{2}$ if and only if $\mathbb{T}_{2}(R)$ is strongly clean (see [Nic99, Example 2] and [BDD07, Theorem 18]).

Corollary 3.15. Let $R$ be a local ring. Then $\mathbb{T}_{2}(R)$ is strongly clean if and only if $\mathbb{T}_{2}(R)$ is optimally clean.

In $\left[\mathrm{DDI}^{+} 13\right]$, the authors investigate strongly clean triangular matrices over abelian rings. In the course of proving that every triangular matrix over a commutative clean ring is strongly clean, they prove a result (see $\left[\mathrm{DDI}^{+} 13\right.$, Lemma 2.4$]$ ) which implies that any commutative clean ring satisfies the hypotheses of Theorem 3.12 . 
Corollary 3.16. Let $R$ be a commutative clean ring. Then $\mathbb{T}_{n}(R)$ is optimally clean for every positive integer $n$.

Remark 3.17. Theorem 3.12, together with the string of corollaries that follow, illustrates why it might be difficult, given the current techniques for constructing strongly clean triangular matrix rings, to find a triangular matrix ring that is strongly clean but not optimally clean. Most existing theorems that show that a triangular matrix ring is strongly clean do so by picking idempotents that satisfy the hypotheses of Theorem 3.12.

There is, however, one recent class of examples which does not follow this exact model. In $\left[\mathrm{DDI}^{+} 13\right.$, Theorem 3.5], the theory of Pierce sheaves is used to prove that certain types of abelian clean rings (called bleached abelian rings) have strongly clean triangular matrix rings. It is not immediately clear whether triangular matrix rings over bleached abelian rings are optimally clean. What is known is that power series over these triangular matrix rings are strongly clean (see [DDI ${ }^{+} 13$, Proposition 3.7]).

We close our study of triangular matrix rings with one final example. We first recall some terminology. Following [Die06], an element $a$ of a ring $R$ is called strongly $\pi$-rad clean if there is an idempotent $e \in R$ with respect to which $a$ is strongly clean and such that $e a^{n} \in J(R)$ for some $n$. A ring is called strongly $\pi$-rad clean if every one of its elements has this property. The class of strongly $\pi$-rad clean rings sits strictly between the strongly $\pi$-regular rings and the strongly clean rings, and such rings occur naturally in a number of applications. For our current purposes, we are interested in the importance of strongly $\pi$-rad clean rings in the study of triangular matrix rings. In [DDI ${ }^{+} 13$, Definition 3.15], the authors define a strongly $\pi$-rad clean ring $R$ to be totally bleached if it has the property that for every $a, b \in R$ and for every pair of idempotents $e, f \in R$ such that $a$ is strongly $\pi$-rad clean with respect to $e$, and $b$ is strongly $\pi$-rad clean with respect to $f$, the map $l_{a}-r_{b}:\left(l_{e}-r_{f}\right)^{2} R \rightarrow\left(l_{e}-r_{f}\right)^{2} R$ is surjective. It is then proved in $\left[\mathrm{DDI}^{+} 13\right.$, Theorem 3.16] that any triangular matrix over a totally bleached strongly $\pi$-rad clean ring is strongly clean (in fact, strongly $\pi$-rad clean).

Note that the totally bleached condition is similar to the $\sigma$-optimally clean condition. The next result, and the remark that follows, clarifies their relationship.

Lemma 3.18. Let $R$ be a totally bleached strongly $\pi$-rad clean ring, and suppose that $\sigma$ is a ring endomorphism of $R$ such that $\sigma(J(R)) \subseteq J(R)$. Then $R$ is $\sigma$-optimally clean.

Proof. Let $a \in R$, and let $e \in R$ be an idempotent with respect to which $a$ is strongly $\pi$-rad clean. Writing $f=1-e$, the totally bleached condition (together with the hypothesis that $J(R)$ is $\sigma$-invariant) ensures that the maps $l_{\text {eae }}-r_{\sigma^{n}(f a f)}: e R \sigma^{n}(f) \rightarrow e R \sigma^{n}(f)$ and $l_{f a f}-r_{\sigma^{n}(e a e)}: f R \sigma^{n}(e) \rightarrow f R \sigma^{n}(e)$ are surjective. Thus $a$ is $\sigma$-optimally clean.

Remark 3.19. It stands to reason that the $\sigma$-optimally clean conditions and the totally bleached condition should look similar, as they are both designed to "lift" strong cleanness in a setting (power series rings and triangular matrix rings, respectively) featuring the completeness of a ring with respect to an ideal. In fact, one could modify Lemma 3.7 and Theorem 3.8 to give analogous results about the totally bleached condition, generalizing $\left[\mathrm{DDI}^{+} 13\right.$, Proposition 4.4].

We note that, for strongly $\pi$-rad clean rings, the optimally clean condition is strictly weaker than the totally bleached condition. In [BDD07, Example 39], there is an example of 
a local ring which is not totally bleached (note that all local rings are strongly $\pi$-rad clean). However, Proposition 3.4 shows that all local rings are skew optimally clean.

In $\left[\mathrm{DDI}^{+} 13\right.$, Theorem 4.5], it is proved, using techniques developed for triangular matrix rings, that if $R$ is a totally bleached strongly $\pi$-rad clean ring and if $\sigma$ is a ring endomorphism of $R$ such that $\sigma(J(R)) \subseteq J(R)$, then $\mathbb{T}_{n}(R[[x ; \sigma]])$ is strongly clean for every $n$. Using Lemma 3.18, we can show that this is a consequence of the ring $\mathbb{T}_{n}(R)$ being $\sigma$-optimally clean.

Theorem 3.20. Suppose that $R$ is a totally bleached strongly $\pi$-rad clean ring, and suppose that $\sigma$ is a ring endomorphism such that $\sigma(J(R)) \subseteq J(R)$. Let $n$ be a positive integer. Extend $\sigma$ componentwise to an endomorphism of $\mathbb{T}_{n}(R)$ in the natural way. Then $\mathbb{T}_{n}(R)$ is $\sigma$-optimally clean.

Proof. It is shown in $\left[\mathrm{DDI}^{+} 13\right.$, Proposition 4.4] that, under these hypotheses, $\mathbb{T}_{n}(R)$ is strongly $\pi$-rad clean and totally bleached. By Lemma 3.18, $\mathbb{T}_{n}(R)$ is thus $\sigma$-optimally clean.

Remark 3.21. Alternatively, the special case of Theorem 3.20 where $\sigma$ is taken to be the identity can be seen as a consequence of Theorem 3.12, since the totally bleached condition implies that the relevant maps are surjective.

We now turn our attention to full matrix rings. We first establish some terminology. If $R$ is a commutative ring, an $R$-algebra will refer to a $\operatorname{ring} S$, together with a ring homomorphism from $R$ into the center of $S$. We will say that an element of $S$ is algebraic over $R$ if it is a root of a monic polynomial over $R$. In [DD13], the authors study the strong cleanness of such elements. We recall the essential results here. Suppose that $R$ is a commutative ring and that $h(t) \in R[t]$ is monic. Following [DD13, Definition 4.3], an $S R C$ factorization of $h$ is a pair of polynomials $f, g \in R[t]$ such that $h=f g,(f, g)=R[t]$, and $f(0), g(1) \in U(R)$. By [DD13, Theorem 4.6], the polynomial $h(t)$ has an SRC factorization if and only if every element $\alpha$ (of every $R$-algebra) which satisfies $h(\alpha)=0$ is strongly clean. For the convenience of the reader, we briefly recall the argument here (for the full details, see [DD13]). An SRC factorization implies that $R[t] /(h) \cong(R[t] /(f)) \times(R[t] /(g))$. Such an element $\alpha$ in an $R$ algebra $S$ is then the image of $t+(h)$ in $R[t] /(h)$, which is strongly clean in $R[t] /(h)$ with respect to the idempotent $(0,1)$ (in the given direct product decomposition). We will now show that the strongly clean decomposition that is implied by an SRC factorization is, in fact, an optimally clean decomposition.

Theorem 3.22. Suppose that $R$ is a commutative ring and that $S$ is an $R$-algebra. Let $\alpha$ be an element of $S$, and suppose that $\alpha$ is a root of the monic polynomial $h(t) \in R[t]$. Suppose further that $h=f g$ is an $S R C$ factorization of $h$, and that $e \in S$ is the corresponding idempotent, with respect to which $\alpha$ is strongly clean. Then $\alpha$ is optimally clean with respect to $e$.

Proof. Write $e^{\prime}=1-e$. By the proof of [DD13, Theorem 4.2], $f\left(e^{\prime} \alpha e^{\prime}\right)=0$ and $g(e \alpha e)=0$. Since $f$ and $g$ are comaximal, there are polynomials $p, q \in R[t]$ such that $f p+g q=1$. Evaluating the polynomials in this equation at $e \alpha e$, and multiplying the result by $e$ (recalling 
that the algebra generated by $R, e$ and $\alpha$ is commutative), we see that $f(e \alpha e) p(e \alpha e) e=e$. Similarly, $g\left(e^{\prime} \alpha e^{\prime}\right) q\left(e^{\prime} \alpha e^{\prime}\right) e^{\prime}=e^{\prime}$.

In order to show that $\alpha$ is optimally clean with respect to $e$, we must show that the maps $l_{e \alpha e}-r_{e^{\prime} \alpha e^{\prime}}: e S e^{\prime} \rightarrow e S e^{\prime}$ and $l_{e^{\prime} \alpha e^{\prime}}-r_{e \alpha e}: e^{\prime} S e \rightarrow e^{\prime} S e$ are surjective. Suppose that $e \beta e^{\prime} \in e S e^{\prime}$. Let $\alpha^{\prime}=\alpha+e \beta e^{\prime}$. Writing out the Peirce decomposition of $\alpha^{\prime}$ with respect to $e$, we get

$$
\alpha^{\prime}=\left(\begin{array}{cc}
e \alpha e & e \beta e^{\prime} \\
0 & e^{\prime} \alpha e^{\prime}
\end{array}\right) .
$$

Since $f\left(e^{\prime} \alpha e^{\prime}\right)=0$, we see that

$$
f\left(\alpha^{\prime}\right)=\left(\begin{array}{cc}
f(e \alpha e) e & e \gamma e^{\prime} \\
0 & 0
\end{array}\right)
$$

for some element $e \gamma e^{\prime} \in e S e^{\prime}$. It is clear that $\alpha^{\prime} f\left(\alpha^{\prime}\right)=f\left(\alpha^{\prime}\right) \alpha^{\prime}$. Looking in the $e S e^{\prime}$ corner, this implies that

$$
(e \alpha e)\left(e \gamma e^{\prime}\right)=f(e \alpha e)\left(e \beta e^{\prime}\right)+\left(e \gamma e^{\prime}\right)\left(e^{\prime} \alpha e^{\prime}\right) .
$$

Left-multiplying by $p(e \alpha e)$ (noting that $p(e \alpha e)$ commutes with $e \alpha e$ ), we see that

$$
e \alpha e\left(p(e \alpha e) e \gamma e^{\prime}\right)-\left(p(e \alpha e) e \gamma e^{\prime}\right) e^{\prime} \alpha e^{\prime}=e \beta e^{\prime},
$$

which shows precisely that $l_{e \alpha e}-r_{e^{\prime} \alpha e^{\prime}}$ is surjective. The surjectivity of $l_{e^{\prime} \alpha e^{\prime}}-r_{e \alpha e}$ is proved analogously. Thus $\alpha$ is optimally clean with respect to $e$.

We thus obtain the following corollary for full matrix rings over commutative rings.

Corollary 3.23. Let $R$ be a commutative ring, and let $n$ be a positive integer. Then $\mathbb{M}_{n}(R)$ is strongly clean if and only if $\mathbb{M}_{n}(R)$ is optimally clean.

Proof. The reverse direction is clear, so we focus on the forward implication. Suppose that $\mathbb{M}_{n}(R)$ is strongly clean. By [DD13, Corollary 4.7], every monic degree $n$ polynomial in $R[t]$ has an SRC factorization. By Theorem 3.22, this implies (following the Cayley-Hamilton Theorem) that every element of $\mathbb{M}_{n}(R)$ is optimally clean.

The following is immediate and subsumes a number of results in the literature (e.g. [CYZ06b, Theorem 9], [FY06, Theorem 2.10], [YZ07, Theorem 2.7]).

Corollary 3.24. Let $R$ be a commutative ring such that $\mathbb{M}_{n}(R)$ is strongly clean. Then $\mathbb{M}_{n}(R[[x]])$ is strongly clean.

Note that our results about optimally clean matrix rings rely heavily on the commutativity of the base ring. In the noncommutative case, substantially less is known about strongly clean matrix rings (see [DD13] for a full discussion). However, in the case of a $2 \times 2$ matrix over a local ring, we can say something.

Proposition 3.25. Let $R$ be a local ring. Then $\mathbb{M}_{2}(R)$ is strongly clean if and only if it is optimally clean. 
Proof. It suffices to prove the forward direction. Suppose that $\mathbb{M}_{2}(R)$ is strongly clean, and choose $A \in \mathbb{M}_{2}(R)$. If either $A$ or $A-I$ is unit, then $A$ is clearly optimally clean, so we may assume that neither $A$ nor $A-I$ is a unit. In that case, $A$ is strongly clean with respect to an idempotent whose kernel and image are both isomorphic to $R$ (since local rings are projective-free). Thus, choosing an appropriate basis, we may assume that $A=\left(\begin{array}{ll}a & 0 \\ 0 & b\end{array}\right)$ where $a \in J(R)$ and $b \in 1-J(R)$ (so that neither $A$ nor $A-I$ is a unit). We claim that $A$ is optimally clean with respect to the idempotent $E=\left(\begin{array}{ll}1 & 0 \\ 0 & 0\end{array}\right)$. We must, therefore, show that $l_{a}-r_{b}: R \rightarrow R$ and $l_{b}-r_{a}$ are surjective. The proof of [Dor06, Proposition 5.1.5] shows that this is indeed the case. Thus, $A$ is optimally clean.

Proposition 3.25 implies immediately that if $R$ is a local ring, then $\mathbb{M}_{2}(R)$ is strongly clean if and only if $\mathbb{M}_{2}(R[[x]])$ is strongly clean (this fact is noted in [Li09, Theorem 3.2]).

The large variety of examples that we have constructed suggests the possibility that every strongly clean ring is optimally clean. Although we do not yet know whether this is true, we can show that in certain situations, a strongly clean power series ring must have an optimally clean base ring. Following [CWZ09], an element is called uniquely strongly clean if it has a unique strongly clean decomposition.

Proposition 3.26. Let $R$ be a ring, and let $a_{0} \in R$ be a uniquely strongly clean element. The following are equivalent.

(1) The element $a_{0}$ is optimally clean.

(2) Any power series of the form $a_{0}+\sum_{i=1}^{\infty} a_{i} x^{i} \in R[[x]]$ is strongly clean.

(3) For every positive integer $n$, any element of the form $a_{0}+a_{1} x+\cdots+a_{n-1} x^{n-1} \in$ $R[x] /\left(x^{n}\right)$ is strongly clean.

(4) Any element of the form $a_{0}+a_{1} x \in R[x] /\left(x^{2}\right)$ is strongly clean.

Proof. Corollary 2.7 shows that (1) implies (2). Since the strongly clean property passes to quotients, (3) follows from (2), and (4) is clearly weaker than (3). It suffices to show that (4) implies (1). Let $e \in R$ be the unique idempotent with respect to which $a_{0}$ is strongly clean. Let $b \in R$ be any element, and consider the element $a_{0}+b x \in R[x] /\left(x^{2}\right)$. By hypothesis, there is an idempotent, necessarily of the form $e+c x$ (since $a_{0}$ is uniquely strongly clean) with respect to which $a_{0}+b x$ is strongly clean. Since $a_{0}+b x$ must commute with $e+c x$, we see that $\left[a_{0}, c\right]=[e, b]$. Thus, $a_{0}$ is optimally clean.

Although we have shown that a wide variety of strongly clean rings are optimally clean, we still do not know whether or not there is an example of a strongly clean ring which is not optimally clean. We pose this formally as a question.

Question 3.27. Is every strongly clean ring optimally clean?

We can construct a strongly clean element which is not optimally clean. The following example relies heavily on [BDD07, Example 45]. 
Example 3.28. Let $k$ be a field. Let $R=k\left[t_{1}, t_{2}, \ldots\right]_{\left(t_{1}\right)}$ be the ring of polynomials in countably many indeterminates over $k$, localized at the prime ideal $\left(t_{1}\right)$. Define $\sigma$ and $\tau$ to be endomorphisms of $R$ which fix $k$ and such that $\sigma\left(t_{i}\right)=t_{i+1}$ and $\tau\left(t_{i}\right)=t_{i+1}+1$ for every $i \geq 1$. Let $A=(R[[x ; \sigma]])[[y ; \tau]]$, a right skew power series ring in $y$ over a left skew power series ring in $x$. Since $R$ is local, so is $A$. Consider the ring $S=\mathbb{T}_{2}(A)$. (It is shown in [BDD07, Example 45] that this ring is not strongly clean.) Let

$$
M_{0}=\left(\begin{array}{cc}
t_{1} & 0 \\
0 & 1+t_{1}
\end{array}\right) \in S .
$$

Since we can view $M_{0}$ as an element of the strongly clean ring $\mathbb{T}_{2}(R)$, it is clear that $M_{0}$ is a strongly clean element of $S$.

We claim that $M_{0}$ is not optimally clean. Note that any strongly clean decomposition of $M_{0}$ must use an idempotent of the form

$$
E_{0}=\left(\begin{array}{ll}
1 & c \\
0 & 0
\end{array}\right)
$$

for some $c \in A$ that satisfies $c=t_{1} c-c t_{1}$. Let

$$
M_{1}=\left(\begin{array}{cc}
0 & x y \\
0 & 0
\end{array}\right) \in S .
$$

Following the same argument as used in [BDD07, Example 45] together with the surrounding discussion (or calculating directly), it can be verified that there is no element $Z \in \mathbb{T}_{2}(A)$ such that $\left[M_{0}, Z\right]=\left[E_{0}, M_{1}\right]$. Therefore, $M_{0}$ is not optimally clean.

We can also use [BDD07, Example 45] to produce a strongly clean ring which is not $\sigma$-optimally clean, for some suitable endomorphism $\sigma$.

Example 3.29. Let $R, \sigma, \tau$ and $S$ be as in Example 3.28. View $S$ as $\left(\mathbb{T}_{2}(R[[y ; \tau]])\right)[[x ; \sigma]]$ (where $\sigma$ has been extended componentwise to an endomorphism of the entire triangular matrix ring in question). By [BDD07, Theorem 41] (applied to right power series rings), $\mathbb{T}_{2}(R[[y ; \tau]])$ is a strongly clean ring. By Corollary $3.15, \mathbb{T}_{2}(R[[y ; \tau]])$ is thus optimally clean. However, since we know that the ring $S$ is not strongly clean, we see that $\mathbb{T}_{2}(R[[y ; \tau]])$ cannot be $\sigma$-optimally clean.

As mentioned in Remark 2.8, we can split Question 3.27 into two sub-questions.

Question 3.30. Let $R$ be a strongly clean ring. Is $R[[x]]$ strongly clean?

Question 3.31. Suppose that $R$ is a ring such that $R[[x]]$ is strongly clean. Must $R$ be optimally clean?

Proposition 3.26 offers some hope for a positive answer to Question 3.31, at least in certain situations. In [BDD07, Definition 31], the authors introduce the notion of an $h$-ring, defined to be a ring with the property that maps of the form $l_{a}-r_{b}$ are injective whenever they are surjective. It is likely that a partial converse to Corollary 2.7 holds under this additional condition (or an appropriate variant thereof). On the other hand, as mentioned 
in Remark 3.17, strongly clean triangular matrix rings over abelian rings might be a good first place to look for a counterexample to Question 3.31.

The next two questions concern other possible examples of optimally clean rings. Since every strongly $\pi$-regular ring is optimally clean (see Proposition 3.5 ), another natural weakening of Question 3.27 is the following.

Question 3.32. Is every strongly $\pi$-rad clean ring optimally clean?

Our final question is motivated by the fact that Proposition 3.26 gives a positive answer to Question 3.31 in the uniquely strongly clean case.

Question 3.33. Is every uniquely strongly clean ring optimally clean?

The authors would like to thank Dr. Warren Wm. McGovern for his time and assistance in completing this paper. The authors would also like to give a hearty thanks to the anonymous referee, whose careful reading and thoughtful suggestions were most appreciated.

\section{References}

[BDD07] Gautam Borooah, Alexander J. Diesl, and Thomas J. Dorsey. Strongly clean triangular matrix rings over local rings. J. Algebra, 312(2):773-797, 2007.

[Che06] Weixing Chen. A question on strongly clean rings. Comm. Algebra, 34(7):23472350, 2006.

[CWZ09] Jianlong Chen, Zhou Wang, and Yiqiang Zhou. Rings in which elements are uniquely the sum of an idempotent and a unit that commute. J. Pure Appl. Algebra, 213(2):215-223, 2009.

[CYZ06a] Jianlong Chen, Xiande Yang, and Yiqiang Zhou. On strongly clean matrix and triangular matrix rings. Comm. Algebra, 34(10):3659-3674, 2006.

[CYZ06b] Jianlong Chen, Xiande Yang, and Yiqiang Zhou. When is the $2 \times 2$ matrix ring over a commutative local ring strongly clean? J. Algebra, 301(1):280-293, 2006.

[CZ07] Jianlong Chen and Yiqiang Zhou. Strongly clean power series rings. Proc. Edinb. Math. Soc. (2), 50(1):73-85, 2007.

[DD13] Alexander J. Diesl and Thomas J. Dorsey. Strongly clean matrices over arbitrary rings. preprint, 2013.

[DDGK12] Alexander J. Diesl, Thomas J. Dorsey, Shelly Garg, and Dinesh Khurana. A note on completeness and strongly clean rings. preprint, 2012.

[DDI $\left.{ }^{+} 13\right]$ Alexander J. Diesl, Thomas J. Dorsey, Wolf Iberkleid, Ramiro LaFuenteRodriguez, and Wm. McGovern, Warren. Strongly clean triangular matrices over abelian rings. preprint, 2013. 
[Die06] Alexander J. Diesl. Classes of strongly clean rings. Ph.D. Dissertation, University of California, Berkeley, 2006.

[Die13] Alexander J. Diesl. Nil clean rings. J. Algebra, 383:197-211, 2013.

[Dor06] Thomas Dorsey. Cleanness and strong cleanness of rings of matrices. Ph.D. dissertation, University of California, Berkeley, 2006.

[FY06] Lingling Fan and Xiande Yang. On strongly clean matrix rings. Glasg. Math. J., 48(3):557-566, 2006.

[Lam01] T. Y. Lam. A first course in noncommutative rings, volume 131 of Graduate Texts in Mathematics. Springer-Verlag, New York, second edition, 2001.

[Li09] Bingjun Li. Strongly clean matrix rings over noncommutative local rings. Bull. Korean Math. Soc., 46(1):71-78, 2009.

[Nic99] W. K. Nicholson. Strongly clean rings and Fitting's lemma. Comm. Algebra, 27(8):3583-3592, 1999.

[NZ04] W. K. Nicholson and Y. Zhou. Rings in which elements are uniquely the sum of an idempotent and a unit. Glasg. Math. J., 46(2):227-236, 2004.

[YZ07] Xiande Yang and Yiqiang Zhou. Some families of strongly clean rings. Linear Algebra Appl., 425(1):119-129, 2007.

Alexander J. Diesl

Department of Mathematics

Wellesley College

Wellesley, MA 02481

USA

Email: adiesl@wellesley.edu

Daniel R. Shifflet

Department of Mathematics

Clarion University of Pennsylvania

Clarion, PA 16214

USA

Email: dshifflet@clarion.edu 